\title{
IDENTIFICACIÓN DELICTUAL JUVENIL: UNA PROPUESTA DE INTERVENCIÓN OCUPACIONAL
}

\section{IDENTIFICACION OFFENDER TEENAGERS: A PROPOSED OCCUPATIONAL INTERVENTION}

\section{María José Poblete A.1}

\begin{abstract}
Resumen
La presente revisión bibliográfica tiene por objetivo entrelazar prácticas de intervención desarrolladas a partir de la puesta en marcha de la Ley de Responsabilidad Penal Adolescente, con los modelos disciplinares de Terapia Ocupacional con el fin de otorgar abordaje integral en este contexto. Al indagar en las historias de vida de los jóvenes privados de libertad nos encontramos con trayectorias de vulneración de derechos impactadas por la segregación social, económica, cultural y ocupacional.

Lo anterior genera identidades ocupacionales marcadas por experiencias con connotaciones negativas por lo que quienes trabajan en el abordaje de estos sujetos deben comprender dichos procesos socioculturales para entregar las herramientas necesarias que contribuyan a la autonomía y al desarrollo de conductas prosociales. Como estrategia desde Terapia ocupacional se visualiza la identificación del joven con una ocupación significativa no asociada a la actividad delictual que favorezca la autonomía, sentido de pertenencia y empoderamiento de los sujetos para su inclusión social.
\end{abstract}

\section{Palabras clave:}

Jóvenes infractores de ley, Identidad ocupacional, Autonomía

\begin{abstract}
Abstrac
The objective of this bibliographic revision is to bring together intervention practices developed out of the enactment of the Adolescent Prison Responsibility Law with disciplinary models of occupational therapy in order to execute an integrated approach in this context/ area. Upon studying/investigating the life histories of encarcerated youth, we discovered trajectories of infringement of rights - affected by social, economic, cultural, and occupational segregation.

The former generates occupational identities characterized by experiences with negative connotations. Thus, people working to approach these subjects should understand these sociocutural processes to give them the tools necessary to contribute to autonomy and the development of positive social conduct. One occupational therapy strategy is to have the young person visualize his/her identification with a meaningful occupation not associated with criminal activity - one that favors autonomy, a sense of belonging, and empowerment for his/her social inclusion.
\end{abstract}

\section{Keywords:}

Teenagers laugh offenders, Occupational identity, Autonomy

Terapeuta Ocupacional. Magíster en Intervención Psicosocial. Diplomado Niñez y Políticas Públicas. Coordinadora Programa Salud Mental Vida Nueva, La Granja.

Fono: 98741561

Código Postal: 8320000

Correo electrónico: mariajose_poblete@yahoo.es 


\section{INTRODUCCIÓN}

Desde inicios del siglo XX nos hemos visto dominados por el proceso globalizador que se encuentra en curso y se mantiene gracias a determinados sectores de la sociedad que lo visualizan como una puerta de entrada a la prosperidad, constituyendo la ideología neoliberal su sustento. Esta última promueve la liberación económica como estrategia para el desarrollo; siendo el mercado su institución central, el cual requiere de la competencia para su subsistencia, manipulando y respondiendo a las necesidades de las personas o creando nuevas necesidades en ellas.

Lo anterior favorece el hecho de que las relaciones de poder que tienen lugar en el modelo económico imperante beneficien a una elite minoritaria y que de forma simultánea marginen seriamente a una inmensa mayoría, impidiendo una efectiva aplicación de la universalidad de los derechos humanos.

Aquel proceso genera exclusión social, limitando la puesta en práctica de los principios de los derechos humanos ya que se focalizan y discriminan, y los sujetos quedan imposibilitados de exigir el ejercicio de sus derechos, debido a que incluso la libertad de poder manifestar esta necesidad se ve restringida. Además se ve coartada la posibilidad que tienen las personas de participar en todas las áreas de su vida y de la sociedad.

Dentro de este contexto, existe un sector de la población que queda mayormente marginado de la protección de sus derechos como seres humanos, en donde el Estado no cumple plenamente con la obligación de velar por que esto ocurra.

Aquel sector lo conforman los jóvenes, principalmente de territorios empobrecidos del país, y en especial me referiré a aquellos que infringen la ley penal. No sólo se encuentran en una condición de vulneración de derechos en el transcurso de su vida, generada por la desigualdad existente, sino que además deben resistir la estigmatización en ejes de "desviación o desadaptación", en donde se les considera como fuente de riesgo o peligro, provocando un proceso de exilio cultural, económico, social y político. Entonces, para construir sus identidades eligen símbolos y signos, decantados por el proceso de globalización, identificándose entre sí y diferenciándose del universo adulto.

En este sentido, en la medida en que haya concordancia entre el logro esperado de realizar una conducta y las condiciones o medios para satisfacerlo, desencadenan "la conducta desviada"; los sujetos que no tie- nen la alternativa que permita la obtención de las metas, tienden a utilizar medios ilegítimos para lograrlas.

Dentro de esto, el delito se configura como la principal actividad de los jóvenes, otorgándole un sentido a sus vidas ya que reafirma una identidad por la cual son reconocidos socialmente y dentro del grupo de pares son validados por el desarrollo de valores, intereses y habilidades en torno a esta ocupación. Por ende, tras una trayectoria de vulneración y "fracasos" en distintos contextos (familiar, comunitario, escolar) es el ambiente delictual la instancia de mayor efectividad a nivel ocupacional y relacional, además de ser el mecanismo de acceso a bienes prioritarios que establece el modelo económico dominante.

Ante eso, el delito es la única actividad que otorga consistencia a sus rutinas diarias, y es que la marginación también se vive en las oportunidades de participación ocupacional. Existe un precario nivel educacional, escasas oportunidades de explorar actividades que otorguen goce y distracción en su tiempo libre, y las posibilidades de empleo se restringen a determinados rubros con escasa estabilidad y bajos salarios.

Frente a la marginación ocupacional mencionada surge la siguiente pregunta: ¿Cuál es el rol del Terapeuta Ocupacional, tanto desde la práctica como en la teoría, en el abordaje con jóvenes infractores de ley de centros privativos de libertad, en el marco del enfoque de derechos?

La revisión bibliográfica busca aportar a la intervención de jóvenes privados de libertad complementando con la disciplina de Terapia Ocupacional. Lo anterior resulta relevante ya que el delito se configura como ocupación principal, siendo limitadas las exploraciones de otras actividades que le otorguen significado a sus vidas. Aspecto a considerar, ante la puesta en práctica de la ley 20.084, con el fin de promover un cuestionamiento continuo en las prácticas realizadas en los programas de tratamiento, rehabilitación y reinserción de los y las adolescentes sancionados por dicha ley.

\section{Jóvenes infractores de ley como sujetos de derechos}

En el escenario sociocultural actual, nos encontramos con niños que sufren día a día diversos tipos de vulneraciones, lo que refleja tanto a nivel macro y micro estructural, la incapacidad de sujetos adultos para responder a las necesidades y de generar contextos de justicia social. Vemos entonces, historias de vida de niños que nos permiten afirmar que sus potencialidades de desarrollo se 
truncaron debido a los entornos desfavorables en donde nacieron, debiendo generar estrategias de supervivencia.

Tal situación se ejemplifica en el entorno más próximo como es el hogar, en donde los niños y niñas son maltratados por personas a quienes aman y en quienes confían, en lugares donde deberían sentirse seguros. La pérdida de confianza y creencia en los seres humanos más cercanos puede provocar sensaciones de temor, suspicacia, inseguridad y aislamiento emocional (Mettifogo \& Sepúlveda, 2005).

De esta manera, vemos sujetos inmersos en un ciclo de violencia en donde el fenómeno se observa como transgeneracional, ya que experimentar violencia en la infancia temprana pudiese aumentar el riesgo de victimización posterior y la acumulación de experiencias violentas replicando constantemente estos hechos.

Entonces las situaciones de violencia, el maltrato y la vulnerabilidad no solo se observan en el ambiente familiar, sino también a nivel escolar y comunitario, evidenciándose una exposición habitual a estas situaciones. Es por esta razón que los niños y niñas que han sufrido abuso sexual, maltrato o recibido trato negligente suelen fugarse frecuentemente del hogar o presentar de forma progresiva socialización de calle, exponiéndose a la explotación, abusos, drogas y delincuencia, aumentando así el nivel de vulnerabilidad.

Ante esto, la reacción de las instituciones sociales y los medios de comunicación es de refuerzo de la estigmatización, de la exclusión o de penalización de los comportamientos de los jóvenes de sectores de escasos recursos, sin considerar las historias de vida ligadas al maltrato y la desprotección. Por ende, el joven que en el proceso de configuración de identidad ve los estigmas, juicios de valor como la actitud y conducta que debe proyectar hacia la sociedad, es lo que se espera de él y lo que lo reafirma como sujeto social.

Pero el panorama se ve mucho más complejo para los jóvenes con historias de vulneraciones y de privaciones, debido a que contempla una sociedad en que para conseguir el éxito como individuo, el dinero es decisivo para el reconocimiento y alcanzar el estatus social deseado. Es así como los jóvenes de sectores de escasos recursos, buscan por diversos medios, entre ellos la actividad delictual, la forma de alcanzar el bien material que los lleva a ser parte de la sociedad en la que están inmersos.

Se establece una forma de consumo opulento con el fin de satisfacer necesidades de construcción de identidad que no se logran construir por otras vías, con una alta preocupación por lo exterior, caracterizado por ves- timentas, aparatos electrónicos y de telefonía, automóviles y armas, identificados como de alto prestigio social por el valor económico que poseen. Por lo tanto, la "fachada" pasa a ser el aspecto que representa la identidad, cayendo en el individualismo y exitismo, donde lo material pasa a ser un bien consagratorio (Moulian, 2002).

El exitismo y la imagen negativa "anti sistema" de los jóvenes con historias de vulneración constituyen al sujeto y la forma en que se vincula con el medio social, generando procesos de exclusión y marginación de todo tipo de oportunidad de bienestar psicosocial.

\section{La ocupación como derecho de participación}

El concepto ocupación se refiere a un término más amplio y complejo que el "hacer" o "estar ocupado", implica el desempeño en las distintas tareas que dan significado a una necesidad tanto individual como colectiva, y que a su vez, otorgan sentido de competencia y satisfacción.

La ocupación humana se basa en la interacción y el significado atribuido a las experiencias sociales que confirman la relación del individuo con el entorno. De esta manera el sujeto es considerado como un ser ocupacional en toda su expresión.

Entonces, describiremos ocupación como acciones aprendidas por medio del proceso de socialización dentro de un contexto, y que le otorga significado y propósito a la vida del sujeto. A través de éstos, se motiva para identificarse con un estilo de vida determinado, en respuesta a lo establecido y valorizado por la cultura, siendo construido socialmente. Mientras mayores sean las posibilidades de acceder y participar en las diversas ocupaciones que la sociedad ofrece, mayor es la posibilidad de desarrollo del sujeto y por ende, mejor será su calidad de vida. Por ejemplo, la oferta educativa para niños y niñas de estratos socioeconómicos bajos es limitada, con escaso apoyo educativo y psicopedagógico para quienes presentan dificultades de comportamiento y de aprendizaje, restringiendo las posibilidades de desarrollo escolar, generando frustración, finalizando en múltiples repitencias y/o deserción escolar.

Los seres humanos presentan una necesidad intrínseca de descubrir, crear, participar y sentirse competentes en su ambiente, lo que emerge de necesidades biológicas y socioculturales. Esto se logra a través del descubrimiento de información, el descubrimiento de nuevos potenciales y la utilización de sus habilidades y capacidades que le otorgan un sentido de control, 
confianza en sí mismo y reafirmación de su bienestar personal.

Por lo tanto, es de vital importancia el acceso a las oportunidades de participación ocupacional ya que permite el desarrollo integral de los niños, niñas y jóvenes con la consecuente adquisición de herramientas necesarias para la toma de decisiones que favorezcan cambios a nivel personal y colectivo, promoviendo de esta forma el proceso de autonomía progresiva.

Pero cuando se produce un quiebre debido a la exclusión que no permite explorar una gama de actividades ni acceder a ocupaciones significativas, se vulnera el derecho de los niños, niñas y jóvenes de participar en todas las áreas de su vida y de la sociedad. Lo anterior limita el desenvolvimiento de los seres humanos como actores sociales influyentes en sus propios cambios y en las decisiones que les afectan, manifestándose situaciones de injusticia social.

De esta manera, hallamos injusticias cuando se excluye, margina o discrimina socialmente a un sujeto de cualquier forma de desarrollo o participación ocupacional, sin tener posibilidades de acceder al patrimonio cultural que forma parte de la sociedad que lo acoge y que favorece el desarrollo del sujeto que no tiene posibilidades de acceder a él; por la situación de inequidad social existente se encuentra con un desarrollo deficiente y en busca de alternativas de mayor alcance de dicho proceso.

Somos espectadores de vidas que sobreviven a las inclemencias del sistema socioeconómico imperante; con escasa posibilidad de explorar-participar en actividades de autocuidado, productivas, de ocio y distracción, y de participación social.

\section{Jóvenes en conflicto con la justicia: la realidad chilena}

Al dejar atrás el modelo tutelar previo a la década de los 90, en donde se debía proteger las condiciones de riesgo del "menor", alejándolo de su entorno y diluyendo la responsabilidad de los hechos delictuales, se establece un estado transicional en lo que a justicia juvenil se refiere, en búsqueda de la concordancia con lo que la Convención de Derechos del Niño plantea. Dicha labor se concreta con la promulgación de la Ley 20.084 de Responsabilidad Penal Adolescente durante el año 2007.

Lo anterior permite generar un sistema de responsabilidad penal especial para los adolescentes entre $14 \mathrm{y}$
18 años, eliminando el "discernimiento", consagrando el derecho a defensa, el debido proceso, a ser oído y a la separación de los adultos. Junto con esto, se instaura un amplio catálogo de sanciones ajustado tanto a la gravedad del delito, como a la edad del imputado, constituyendo la privación de libertad como último recurso, contexto al que me referiré en el desarrollo de la presente.

Las cárceles se caracterizan por ser espacios represivos, con un permanente control no sólo de la libertad del sujeto sino que también de sus actos, hábitos, rutinas, intereses, de la expresión de sus ideas, y emociones, e incluso de su autonomía y de sus derechos como seres humanos. "Existe una coacción calculada que recorre cada una de las partes del cuerpo, dominándolo y volviéndolo disponible, generando de esta manera automatismos de los hábitos; llegando incluso a la manipulación de su cuerpo, sus gestos y su comportamiento" (Foucault, 1975).

Lo descrito no sólo guarda relación directa y absoluta para unidades penales de adultos sino que configura también lo que los jóvenes privados de libertad en Chile viven, más allá de los requerimientos y orientaciones técnicas que se plantean en las "Reglas de las Naciones Unidas para la protección de los menores privados de libertad". Los sujetos reafirman que se encuentran "presos", al igual que los adultos en la misma situación.

Las condiciones materiales tales como el hacinamiento, la infraestructura deteriorada e inadecuada para un propósito rehabilitador, conviven con un clima tenso, violento y alienante (Selander, 2008).

Se dispuso que el Servicio Nacional de Menores sería la entidad encargada de ejecutar todas las acciones y programas tendientes a hacer efectiva dicha responsabilidad en forma especializada. Y es quien ha establecido orientaciones técnicas en este sentido.

En la oferta programática se distinguen niveles de intervención, los cuales son educación, tiempo libre y recreación; familia e integración social; y necesidades especiales como salud mental, drogas, identidad delictual y desarrollo laboral. Estos niveles buscan cumplir con lo que la ley propone como objetivo de hacer efectiva la responsabilidad de los adolescentes, de tal manera que la sanción forme parte de una intervención socioeducativa amplia y orientada a la plena integración social. Según este objetivo los niveles de intervención se detallan de la siguiente manera (SENAME, 2007).

A nivel educativo, existen tres procesos de integración escolar: nivelación de pre ingreso a escuela, para aquellos/as adolescentes que requieren de atención individualizada por presentar descensos importantes en 
área instrumental (Lecto-Escritura y Cálculo), área cognitiva y en nivel de adaptación escolar.

En segundo lugar, el ingreso a la escuela con acompañamiento, para aquellos/as jóvenes que presentan descensos moderados en área instrumental, área cognitiva y en niveles de adaptación escolar.

Finalmente, el ingreso a la escuela de forma autónoma, para aquellos/as jóvenes que se encuentran debidamente escolarizados, es decir, que no presentan deserción escolar a su llegada al centro, y sólo con leves dificultades de aprendizaje. Además existen talleres de educación no formal en las líneas de artes escénicas, plásticas, audiovisuales, musicales; actividades deportivas y culturales.

Los talleres en general deben considerar normas de convivencia, objetivos claros que se relacionen con los objetivos de la intervención en general, fomento de la autoeficacia, desarrollo de creatividad y capacidades cognitivas.

En las actividades recreativas se distinguen las estructuradas y el tiempo o espacio libre para la recreación. De esta manera, se debe entregar herramientas a los jóvenes para que logren organizar sus períodos de ocio, sin quedarse absolutamente desocupados.

El proceso de reinserción social supone un trabajo y vinculación con sus familias que favorezca un mejoramiento de las relaciones entre sus miembros, que fortalezca los proyectos de vida de cada joven y que asegure un proceso de reinserción social y familiar.

En el ámbito laboral, se realizan entrevistas vocacionales que determinen intereses y disposición a la capacitación y el trabajo. Entrenamiento intensivo y acceso al empleo productivo en adolescentes que han participado de los programas de formación laboral.

En tanto, lo que respecta a las necesidades de salud mental y tratamiento de adicciones se abordan por medio de programas específicos.

La finalidad de los distintos programas mencionados es que se centren en el individuo, en la familia y en la comunidad para abordar de manera eficaz los factores de riesgo dinámicos de reincidencia delictual.

\section{Reinserción tras las rejas}

Si bien la Ley penal juvenil enfatiza la responsabilización por medio de la relevancia de la reinserción, normalización educativa y capacitación laboral, como también la rehabilitación de las adicciones de los jóvenes que sean imputados de un delito, si ello es reque- rido. Una vez definida la sanción, el énfasis que se intenta en la intervención, es de carácter socioeducativo en la perspectiva de la reinserción social. Ello implica una intervención que asegure un acompañamiento global al adolescente en su calidad de sujeto en desarroIlo, enfatizando explícitamente una lógica de relación educativa adecuada a las necesidades y recursos del adolescente así como también a las características y condiciones del contexto en el que él se desarrolla (SENAME, 2007).

En la actualidad se puede apreciar en los medios cerrados, que están operando bajo la lógica de un modelo de control que genera condiciones que refuerzan la prisionización. Se aprecia un esfuerzo sostenido en garantizar la seguridad estática (presencia de gendarmería, centrado en el control), pero poco respecto de la seguridad dinámica, la que se relaciona con un modelo relacional y prosocial, ello es: normas claras, capacidad para contener, preocupación por la equidad con el consiguiente esfuerzo para evitar luchas y abuso de poder, énfasis en los valores de la convivencia, todo lo cual exige la presencia de equipos competentes además de condiciones de arquitectura apropiadas a una labor educativa (Dionne \& Zambrano Constanzo, 2009).

Entonces hablar de intervención y reinserción social en contextos de reclusión es complejo, y más aún es el ejercicio de derechos ya que el sujeto se ve dominado en los significados y construcciones que han conformado su identidad, no solo por el control social que se ejerce en su máxima expresión sino que también por el quiebre que se intenta realizar por medio de la intervención al estilo de vida que practican. Permanecen bajo esta dinámica de control la cual finalmente logra que el sujeto se mantenga bajo dicha estructura y se autocontrole en base a la norma establecida como régimen interno.

Por otro lado, resulta complejo para el sujeto alcanzar la participación social plena y el consiguiente empoderamiento, si se ha sentido siempre fuera del sistema social en el cual se encuentra inmerso, y en lo que respecta a la vida ocupacional no ha sido significativa por las dificultades para acceder a las oportunidades de protección y desarrollo. Es fundamental que los sujetos logren un sentido de pertenencia, entendiendo que las razones de la exclusión social de sí mismo es parte del proceso de sentirse incluido.

Debido a estas razones la labor interventiva en ambientes de privación de libertad se torna ambiciosa, es un terreno hostil marcado por juicios y discriminación, y por un sistema económico, social y político que no 
logra abordar la problemática de la delincuencia juvenil desde sus causas directas, otorgando respuestas paliativas.

Es entonces, un desafío para los equipos profesionales realizar un trabajo satisfactorio y que no provoque frustración. Por este motivo quienes trabajan directamente con los jóvenes privados de libertad deben ser capaces de generar en conjunto con el sujeto de intervención metas realistas y considerando que las intervenciones del medio cerrado son un elemento más de todo el proceso de reinserción social.

\section{Identidades juveniles}

En relación a la identidad delictual me basaré en aspectos que considero fundamental abordar desde la perspectiva de Terapia Ocupacional y que permitirían generar la motivación y disposición por el cambio de forma intrínseca y no debido a factores externos como la adaptación a lo que socialmente está impuesto.

\section{Definición de una ocupación significativa}

El principal factor de desarrollo personal corresponde a la apropiación de la experiencia social y especialmente del patrimonio cultural, por ende, se entiende que las desigualdades del desarrollo se relacionan directamente con las facilidades para acceder a esa experiencia. Esta limitante se observa también con la gama de roles y de desempeños en ocupaciones que sean adquiribles por medio de las relaciones sociales, por lo que a mayor carencia socioeconómica, mayor es la restricción para participar en una variedad y diversidad de actividades. Tal es el ejemplo de las actividades de tipo recreativos que por lo general se acotan a actividades deportivas, principalmente el fútbol, sin tener posibilidades de acceder a una oferta de instancias de esparcimiento más amplia con la factibilidad de realizar elecciones ocupacionales y no acotarse a lo que existe de forma restringida.

Cuando se habla de ocupación se refiere no solo al ámbito laboral sino que también a lo educativo, al tiempo libre y a instancias de autocuidado, por lo que se hace necesario que los niños, niñas y jóvenes tengan la oportunidad de conocer distintas disciplinas para estas áreas. Entonces la exploración ocupacional se constituye como forma de participación e intercambio de patrimonio cultural.
Al definir una o varias ocupaciones significativas se configura la identidad del sujeto. La identidad ocupacional emerge en la infancia, a medida que los niños adquieren la capacidad para integrar pasado, presente y futuro e imaginarse en una historia que se despliega, comienzan a narrar parte de sus vidas y a seleccionar significados a través de las historias (Kielhofner, 2004).

Por esta razón es de especial cuidado el confrontar al joven en relación a la participación en la actividad delictual de los jóvenes privados de libertad ya que es una de las pocas alternativas que se les ha otorgado tanto como actividad productiva, recreativa y de autocuidado que le dé sentido a sus vidas y se sientan efectivos en el entorno.

Al plantear las intervenciones se debe tener precaución de que las actividades que se establezcan junto al joven no se perciban como impuestas y se observe que son parte de un proceso de exploración y de consideración de las preferencias, disposición al cambio y voluntad del sujeto, por lo que las actividades no son para "matar el tiempo" ni "hacer por hacer" sino que para dar inicio a un proceso en que se les brinde sentido a sus vidas por medio de ocupaciones que resulten de la motivación intrínseca y no desde la obligatoriedad.

El Modelo de Ocupación Humana desarrollado por Gary Kielhofner plantea que existen tres etapas en el cambio ocupacional que describe ampliamente las trayectorias que pueden ser adoptables en este proceso:

La exploración es la primera etapa de cambio en la cual las personas intentan nuevas cosas y en consecuencia aprenden de sus propias capacidades, preferencias y valores. Las personas exploran cuando están aprendiendo nuevas formas ocupacionales, haciendo cambios de roles o buscando nuevas fuentes de significado. La exploración brinda la oportunidad para el aprendizaje, el descubrimiento de nuevos modos de hacer y el descubrimiento de nuevas formas de expresar capacidad y aprender la vida. La exploración requiere de un ambiente relativamente seguro y poco demandante, dado que una persona que está explorando aún no está segura acerca de la capacidad o el deseo. Los recursos y las oportunidades en el medio ambiente son críticos. Las ocupaciones exploradas deben mostrar a los adolescentes la existencia de oportunidades de participación ocupacional, distintas a la actividad delictual.

La competencia es la etapa en que las personas comienzan a solidificar nuevas formas de hacer que fueron descubiertas a través de la exploración. Se ajustan a las demandas y expectativas ambientales. Se concentran en un desempeño compatible y adecuado. Con- 
duce al desarrollo de nuevas habilidades, refinamiento de las antiguas y la organización de estas habilidades en hábitos que sustenten el rendimiento ocupacional. Otorga un sentido creciente de control personal. A medida que las personas luchan por organizar sus desempeños en rutinas de comportamientos competentes que son relevantes para su medio ambiente, se sumergen en un proceso de convertirse, crecer y llegar a un mayor sentido de eficacia. En esta etapa es donde comienza la problematización de la actividad delictual, en donde se sentían eficaces en su desempeño, con la nueva ocupación explorada.

En la etapa de logro, las personas tienen suficientes habilidades y hábitos, los que les permiten participar plenamente en algún trabajo, actividad recreativa o de las vidas diarias nuevas. Integra una nueva área de participación ocupacional en su vida total, por lo que la identidad ocupacional es remodelada para incorporar el área nueva de participación ocupacional. Otros roles y rutinas deben alterarse para acomodar el nuevo patrón global para sostener la competencia ocupacional y en este sentido construir proyectos ocupacionales acordes a este proceso de cambio.

En la intervención con jóvenes infractores, el paso por las etapas de cambio que faciliten la participación en una nueva ocupación que se constituya en significativa permitirían ir modificando y adecuando sus estilos de vida a las nuevas preferencias, lo que genera la ambivalencia de dar continuidad al desempeño delictual.

\section{Participación social y sentido colectivo}

El hecho de participar en actividades delictuales implica pautas relacionales que se potencian con el modelo económico dominante. Debido a esta razón vemos jóvenes que se caracterizan por la búsqueda del éxito, inmediatismo, competencia e individualismo.

Las intervenciones deben contemplar el abordaje de estas formas de interacción, tras la comprensión de los procesos sociales. Una estrategia que facilita lo anterior es la generación de espacios grupales con los jóvenes que fomenten la creación de proyectos colectivos, con replicabilidad en contextos comunitarios en donde habitan. Dichos proyectos debiesen potenciar el trabajo en equipo por sobre la competitividad y el individualismo. Dicha propuesta favorecería el desarrollo de distintos componentes del desempeño ocupacional:
- La adquisición de roles dentro de un equipo involucra asumir responsabilidades y compromisos hacia un objetivo común.

- Se favorece el aprendizaje en conjunto y el conocimiento de nuevas experiencias y formas de hacer, promoviendo simultáneamente la creatividad.

- Es necesario poner énfasis en los procesos que permiten alcanzar el resultado final, considerando obstáculos y facilitadores que pueden determinar variaciones en los tiempos estimados. Lo anterior permite vivenciar el éxito y fracaso como experiencias válidas.

- Cuando los proyectos son autogestionados producen mayor sentido de eficacia en los sujetos y mayor valoración de los esfuerzos desplegados.

Con esta forma de abordaje, implícitamente se intervienen de forma individual y grupal aspectos psicosociales dinámicos asociados a la delincuencia, tales como la asociación a pares infractores, identidad delictual, resolución de conflictos, comunicación efectiva, control de impulsos, entre otros. Estas instancias permiten generar la autonomía grupal e individual, siendo el Terapeuta Ocupacional un facilitador de procesos.

\section{CONCLUSIONES}

En Chile hay muy pocos reportes acerca de experiencias en el ámbito de la rehabilitación con jóvenes infractores de ley, existiendo escasa evidencia de evaluaciones sistemáticas a través del tiempo, siendo más limitada la información relacionada con la intervención en un régimen cerrado.

Según lo expuesto resulta contradictorio que este joven que ha vulnerado a sus víctimas Ilegue a la instancia de la prisión pensando en que él ha tenido una historia de vida marcada por las vulneraciones de sus derechos como niño/joven, por parte de otros sujetos que no reciben sanción. Lo anterior muestra la relevancia de un abordaje previo de tipo promocional y preventivo que fortalezca los garantes de la infancia y la juventud a favor del desarrollo integral. No obstante, la privación de libertad es una realidad, la cual debe ser abordada, debiendo cubrir necesidades individuales, 
familiares y comunitarias con un equipo profesional diverso que pueda apoyar la inclusión de los jóvenes.

Dentro de esta instancia, el Terapeuta Ocupacional puede ser un aporte en el proceso de inclusión ya que los jóvenes llegan a la reclusión por el desempeño de una ocupación como lo es la actividad delictual, la cual se constituiría en significativa dentro de su historia vital ya que otorga identidad, sentido de eficacia y percepción de logro. Lo anterior nos permite reafirmar que "La actividad pasa a ser significativa cuando se entrelazan varias significaciones caleidoscópicas: es aprendida, vivida y realizada por cada uno de sus actores y se modifica por la intención de transformación existente en los objetivos del programa donde se da la actividad" (Kronenberg, Simó Algado \& Pollard, 2006).

La idea es que a través del uso terapéutico de las ocupaciones se generen procesos reflexivos que favorezcan la problematización de las prácticas delictuales para que posteriormente el joven comprenda y establezca estrategias de afrontamiento, toma de decisiones y resolución de conflictos no solo a nivel individual sino que también a nivel colectivo; lo anterior involucra el análisis de los costos y beneficios de la mantención de la conducta infractora o de la búsqueda de un nuevo estilo de vida.

Como Terapeutas Ocupacionales debemos generar en los sujetos la idea de que son ellos los promotores de las oportunidades de participación ocupacional con el consecuente empoderamiento social, con habilidades para la resolución de problemas que se afrontan en la vida diaria a nivel comunitario, capaces de generar transformaciones sociales y concientización de su entorno más próximo.

En este sentido, el Terapeuta Ocupacional adquiere un rol político, ya que debe fomentar la justicia ocupacional, promoviendo la igualdad de condiciones para la exploración de ocupaciones. Lo anterior expresa preocupaciones éticas, morales y cívicas en la vida diaria y debería contribuir a la salud, al empoderamiento y a la calidad de vida de los sujetos.

\section{RefERENCIAS BibliográfICAS}

Dionne, J. \& Zambrano Constanzo, A. (2009). Intervención con adolescentes infractores de ley. El Observador.

Duarte, C. (2001). ¿Juventud o Juventudes? Versiones, trampas, pistas y ejes para acercarnos progresivamente a los mundos juveniles. PASOS.

Foucault, M. (1975). Vigilar y Castigar. Nacimiento de la prisión. París: Siglo XXI.

Kielhofner, G. (2004). Terapia Ocupacional. Modelo de Ocupación Humana, Teoría y aplicación. Madrid: Panamericana.

Kronenberg, F.; Simó Algado, S. \& Pollard, N. (2006). Terapia Ocupacional sin Fronteras, aprendiendo del espíritu de supervivientes. Madrid: Panamericana.

Mettifogo, D. \& Sepúlveda, R. (2005). Trayectorias de vida de jóvenes infractores de ley. Instituto de Asuntos Públicos. Santiago: Universidad de Chile.

Moulian, T. (2002). Chile actual: Anatomía de un mito. Santiago: LOM.

Pinheiro, P. (2006). Informe a Naciones Unidas de expertos sobre la violencia contra los niños y niñas. Panamá.

Selander, M. (2008). Derechos y compromisos: En busca de un modelo pedagógico para jóvenes privados de libertad. Sistematización del programa de re escolarización en un centro cerrado de SENAME. Santiago: ACHNU.

SENAME (Abril de 2007). Ministerio de Justicia. Obtenido de http:// www.sename.cl/wsename/otros/rpa/ResEx0184B_cerrados. pdf

Vidal, M. (1988). El Hombre Inconcluso: desarrollo personal y clase social. Santiago: Cintras. 